\title{
Rapid Progression of Pott's Disease after Short Term Anti- Tuberculosis Drug Discontinuation for Regimen Adjustment: Case Report
}

\author{
Dong Wook Kim ${ }^{1}$, Chang Hwa Ham ${ }^{1,2}$, Jung Hyun $\mathrm{Na}^{1}$, Seung Hoon Lee', \\ Hong Joo Moon', Joo Han Kim ', Youn-Kwan Park', Woo-Keun Kwon ${ }^{1,2}$
}

'Department of Neurosurgery, Korea University Guro Hospital, Korea University College of Medicine, Seoul, Korea

${ }^{2}$ Focused Training Center for Trauma, Korea University Guro Hospital, Korea University College of Medicine, Seoul, Korea

We present a case of spinal tuberculosis (TB) whom showed significant progression of imaging findings as well as neurologic deterioration after short term discontinuation of anti-tuberculosis medication. A 26-year-old man without any clinical past history of tuberculosis complained only back pain and subtle numbness at his leg. After proper diagnosis was made based on laboratory findings by biopsy, anti TB medication was started following the recommended guidelines. However, due to drug rash at the day of medication initiation, anti TB regimen were hold for a brief duration which was less than 3 days. After that, although we were adding on TB medications step by step the patient revisited our emergency room with sudden onset of incomplete paraplegia due to prominent progression of epidural abscess and cord compression. While the progression of spinal TB is known to be insidious and gradual in most cases, this patient presented both clinical and radiographic significant progression within 2 weeks. Anti-tuberculosis medication has key role in treatment of spinal tuberculosis just like any other tuberculosis. We suggest that when discontinuing anti TB medication in the active phase of disease, extra caution must be given.

Key Words: Pott's disease; Tuberculosis spondylitis; Rapid progression

\section{INTRODUCTION}

Spinal tuberculosis(TB), also known as Pott's disease, occurs in less than $1 \%$ of patients with $\mathrm{TB}$, but it is known to be about

Received: June 6, 2021 Revised: June 23, 2021 Accepted: June 25, 2021 Corresponding Author: Woo-Keun Kwon

Department of Neurosurgery, Korea University Guro Hospital, Korea University College of Medicine, 148 Gurodong-ro, Guro-gu, Seoul 08308, Korea.

Tel: +82-2-2626-3100, Fax: +82-2-863-1684, E-mail: kwontym@gmail.com ORCID: https://orcid.org/0000-0003-0432-8620 half of skeletal TB and the most dangerous form of skeletal TB [1-4]. TB is known to affect more than 10 million people worldwide, and approximately 150,000 new patients are being diagnosed as spinal TB annually [5]. Just like the insidious nature of TB, the onset and progression of spinal TB is also insidious that its gradual progression makes varied clinical presentation and occasionally become an obstacle to diagnosis $[2,6,7]$. In most cases, TB spondylitis Multi regimen of anti-tuberculosis drug is a standard treatment of spinal TB and it is the essential part of treatment [8-10]. Majority of patients whom receive proper medical care, are likely to present a favorable outcome

(C) 2021 Korean Neuro-Pain Society

(c) This is an Open Access article distributed under the terms of the Creative Commons Attribution Non-Commercial License (http://creativecommons.org/licenses/by-nc/4.0/) which permits unrestricted non-commercial use, distribution, and reproduction in any medium, provided the original work is properly cited. 
$[4,5,7,11]$, however some do experience uncontrolled prognosis or deterioration in terms of neurologic or radiologic outcomes [4]. In this report, we present a rare case of remarkably rapid progression after short term discontinuation of anti TB drugs.

\section{CASE REPORT}

A 26-year-old man without significant clinical history visited to our center suffering from back pain and numbness along to both calves. Symptoms suddenly began 2 months before and got aggravated regardless of treatment. The patient reported that he had recently lost about $4 \mathrm{~kg}$ of body weight. On initial thoracolumbar spine MRI with contrast enhancement, multifocal heterogeneous bone destructive lesion with strong heterogeneous enhancement at T7, 10, 11, L4 and S2 to S4 levels were
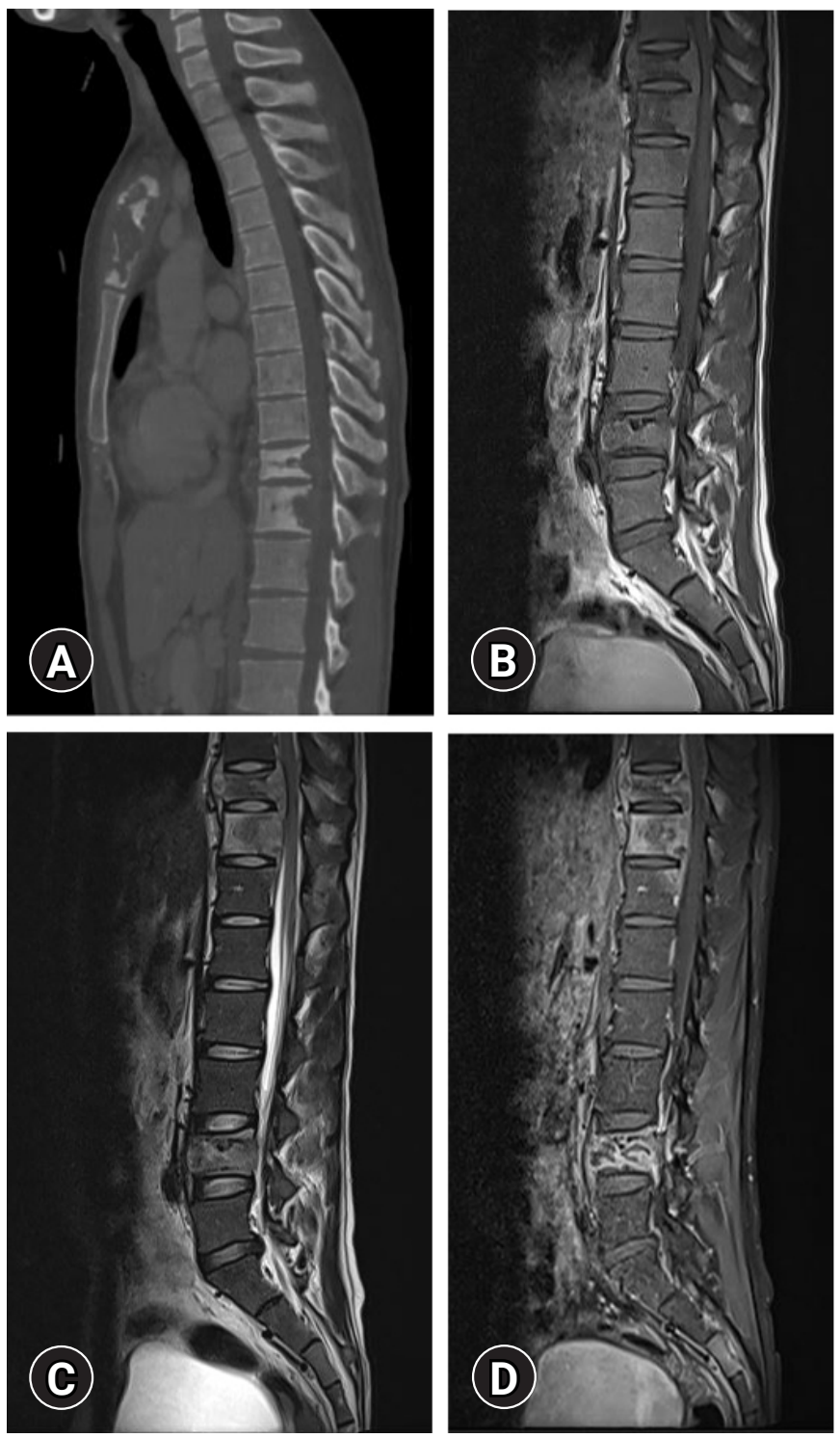

noted. Generalized bone marrow signal alteration was noted at both T1 and T2 weighted images to low signal intensity. Multilobulated enhancing mass at intraosseous, anterior epidural space and paraspinal space were noted. And subtle spinal cord compression was suspected at T10 and T11 level by epidural enhancing mass (Fig. 1). Significantly elevated C-reactive protein (CRP) was also examined (126.74 mg/L). According to these results, the most suspicious finding was tuberculosis, but it needed to be distinguished from the malignancy such as bone metastasis or other conditions. Chest computed tomography (CT) scan was performed and evidence of pulmonary tuberculosis (TB) did not exist but we found cortical destruction of sternal manubrium. We performed needle biopsy from sternal lesion and L4 body. Pathologic examination reported only chronic granulomatous inflammation with necrosis but no evidence of caseous necrosis but mycobacterium tuberculosis (MTB) DNA real time PCR test was positive. Therefore, we finally diagnosed as multi-level spinal tuberculosis. We decided to start anti-tuberculous medication including ethambutol, isoniazid, pyridoxine, pyrazinamide, rifampicin according to the guideline as soon as the diagnosis was made [8]. At that time, neurological symptoms were only back pain and subtle numbness, so surgical treatment was not necessary. 12 hours after the dose, he showed a sudden rash and complained itching sensa-

Fig. 1. Initial thoracolumbar computed tomography (CT) and magnetic resonance image (MRI). (A) CT: Diffuse osteolytic lesion was noted at T10, 11 body. (B) Sagittal view of MRI T1 weighted image (T1WI). (C) Sagittal view of MRI T2 weighted image (T2WI). (D) Sagittal view of MRI T1WI with contrast enhancement. (E) Axial T1WI with contrast enhancement at T10/11 level.

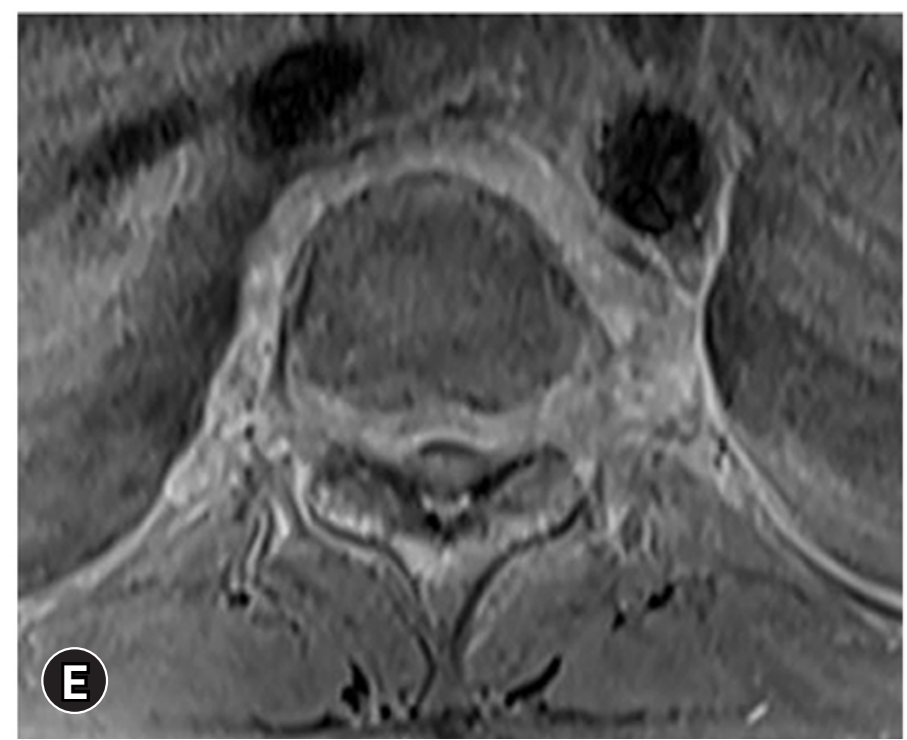


tion all over his body. It was highly suspected of side effect of anti-tuberculosis drugs, so medication was stopped following recommendations from the department of infectious internal medicine. Stepwise add on of drug by drug was every 2 days and the patient was discharged, planning to adjust the medication on an out-patient department basis. At this time point the patient was under three TB medications (pyridoxine, isoniazid and rifampin) However, 3 days after discharge, he came back to emergency room with sudden onset of incomplete paraplegia. Bilateral lower motor weakness was noted and the motor grade was 2 from hip flexion to ankle dorsiflexion. Spine MRI was immediately performed and cord compression was significantly progressed by enlarged epidural enhancing mass suggesting significant progression of spondylitis at T9-12 level (Fig. 2). The amount of paraspinal and epidural abscess surrounding the T10 vertebra body was significantly increased when compared to previous MRI which performed about 3 weeks ago.
The CRP level was peaked up to $210.87 \mathrm{mg} / \mathrm{L}$ indicating uncontrolled progression of the infectious condition. Emergent operation was done for decompression of cord as well as restoration of the spinal alignment. Total laminectomy and corpectomy of T10, 11 was performed and interbody mesh cage was inserted following pedicle screw fixation (Fig. 3). Yellowish, necrotic tissue mixture with pus like fluid was noted in the vertebral body and pathologic exam reported granulomatous tissue with caseous necrosis. The patient's muscle strength had improved since surgery and underwent rehabilitation.

\section{DISCUSSION}

TB spondylitis (Pott's disease) is most common and dangerous form of skeletal TB $[1,2,12,13]$. Incidence of neurological complication in TB spondylitis is $10 \%$ to $20 \%$ in highly developed nations and $20 \%$ to $41 \%$ in underdeveloped countries,
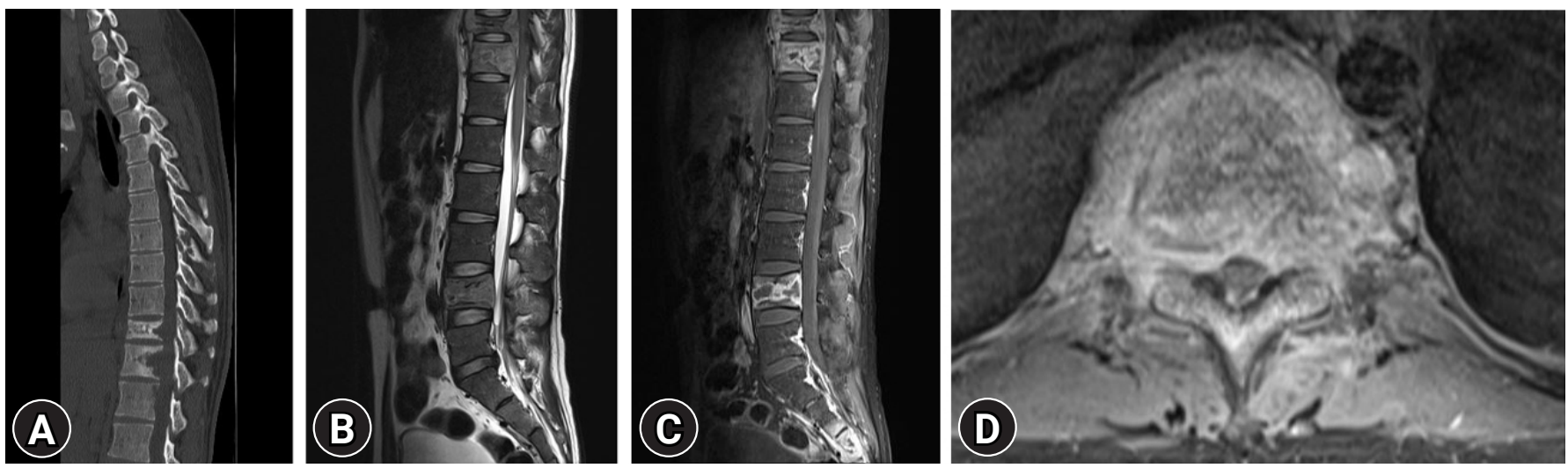

Fig. 2. CT and MRI which performed when patient revisited emergency room with incomplete paraplegia. (A) CT: Osteolytic lesion of T1011 body was extended and the height of T10 body slightly decreased compared to previous CT (Fig. 1A). (B) Sagittal view of T2WI. (C) Sagittal view of MRI T1WI with contrast enhancement. (D) Axial T1WI with contrast enhancement at T10/11 level: Enhancing lesion was definitely extended compared to previous MRI (Fig. 1E).
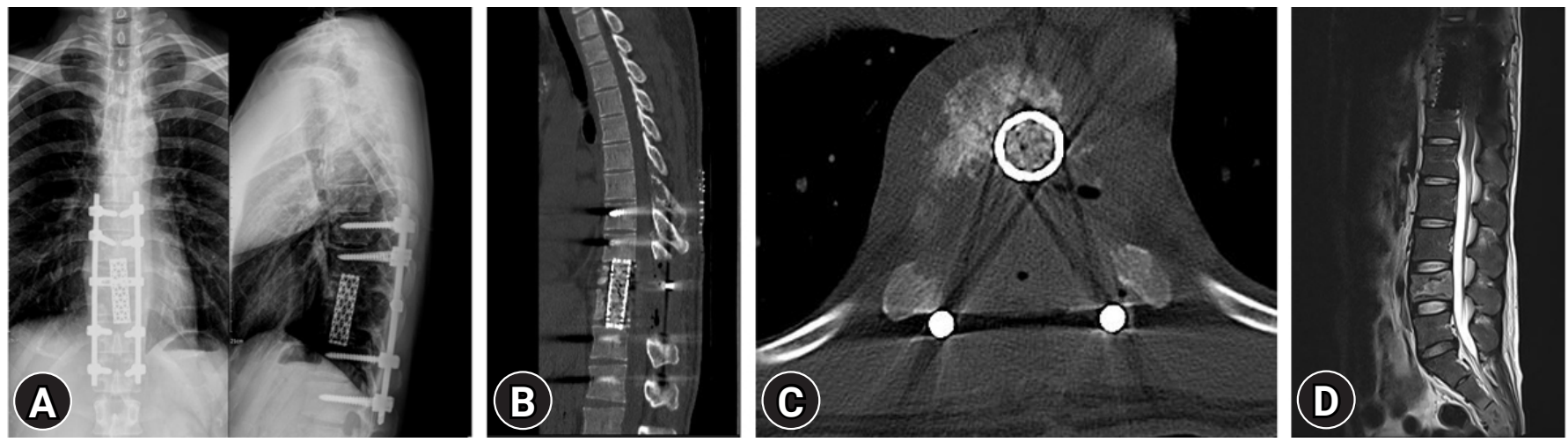

Fig. 3. Postoperative X-ray, CT, MRI. (A) X-ray image AP/LAT. (B) Sagittal image of CT. (C) Axial image of CT at T10/11: Total laminectomy and partial corpectomy was performed. (D) Sagittal image of MRI T2WI. 
particularly if thoracic spine is involved [14]. Surgical intervention is needed if patient has neurologic deficit but anti-tuberculosis medication is essential part of treating TB spondylitis [8,9,15-17]. Therefore, those without significant and distinctive factors which meet the criteria for surgery are of subject for medical treatment $[4,8,9]$. Standard chemotherapy of TB is 2 months for initial intensive phase and 4 months for maintenance therapy. Multidrug therapy is recommended with isoniazid, ethambutol, pyrazinamide, rifampicin [8]. Most patient response relatively good to this specific regimen, however there are certain patients whom present side effects $[10,16,18]$. Any type of anti-tuberculosis drug can occur various degrees and types of side effects starting from simple rashes or itching up to those including life-threatening drug reactions like Steven-Johnson syndrome or DRESS syndrome $[16,18,19]$.

This patient has suffered rash and itching sensation of whole body right after taking medication. He did not have any other symptoms like petechia or fever which are more strongly suspicious symptoms of serious drug reactions but we stopped medication due to considering risk and irreversibility of severe side effect. Guideline said that in case of patient getting skin side effects, it should be to temporarily administer three different anti-tuberculosis drugs while discontinuing their existing medications if patient is considered to be with severe tuberculosis [8]. But in our case, neurological deficit or severity of spondylitis was not that severe which exceed risk of life-threatening side effects at that moment. In most case, when anti-tuberculosis drugs are hold temporarily, re-dosing one drug by another every two to three days does not take that big risk for the patient. It takes about two weeks in total to re-take all four medications or other suitable alternative combinations. Moreover, TB spondylitis is known to have slow and insidious progression, so it was not easy to predict such a devastating rapid progression such as in our case. Although we followed the recommendation of guidelines for anti-TB medication selection, and also that for medication adjustment in cases of side effects, the consequent result was not what we expected.

This case has been relatively rare in many aspects, including its non-contiguous feature since most TB spondylitis has contiguous lesion $[3,20,21]$. It should be considered and kept in mind that TB spondylitis, especially those presenting with atypical clinical features can result as an unexpectedly fast progression of disease and also spinal cord damage in a short period of time. It means that the possibility of acute progression is never negligible in this disease. Even if you see side effects of anti-tuberculosis drugs, if the symptoms are adjustable and mild, we sjuggest that medication is better to be continued with symptom relief medications like anti-histamine drugs together [11]. If it is difficult, at least another anti tuberculosis medication should be taken equivalent to severe tuberculosis as the guideline says [11]. Delayed anti- tuberculosis medications can cause critical neurological injury and sometimes vigilant approach is needed in TB spondylitis.

\section{CONFLICTS OF INTEREST}

No potential conflict of interest relevant to this article was reported.

\section{REFERENCES}

1. Alothman A, Memish ZA, Awada A, Al-Mahmood S, Al-Sadoon S, Rahman MM, et al.. Tuberculous spondylitis: analysis of 69 cases from Saudi Arabia. Spine (Phila Pa 1976) 2001;26:E565-E570

2. Nussbaum ES, Rockswold GL, Bergman TA, Erickson DL, Seljeskog EL. Spinal tuberculosis: a diagnostic and management challenge. J Neurosurg 1995;83:243-247

3. Kulali A, Cobanoğlu S, Ozyilmaz F. Spinal tuberculosis with circumferential involvement of two noncontiguous isolated vertebral levels: case report. Neurosurgery 1994;35:11541158

4. Dai LY, Jiang LS, Wang W, Cui YM. Single-stage anterior autogenous bone grafting and instrumentation in the surgical management of spinal tuberculosis. Spine (Phila Pa 1976) 2005;30:2342-2349

5. Pawar UM, Kundnani V, Agashe V, Nene A, Nene A. Multidrug-resistant tuberculosis of the spine: is it the beginning of the end? A study of twenty-five culture proven multidrug-resistant tuberculosis spine patients. Spine (Phila Pa 1976) 2009;34:E806-E810

6. Garg RK, Somvanshi DS. Spinal tuberculosis: a review. J Spinal Cord Med 2011;34:440-454

7. Rajasekaran S, Soundararajan DCR, Shetty AP, Kanna RM. Spinal tuberculosis: current concepts. Global Spine J 2018; 8:96S-108S

8. The Korean Academy of Tuberculosis and Respiratory Diseases, Korea Disease Control and Prevention Agency. Korean guidelines for tuberculosis. 4th ed. Cheongju: Korea Disease Control and Prevention Agency; 2020

9. Moon MS. Tuberculosis of the spine. Controversies and a new challenge. Spine (Phila Pa 1976) 1997;22:1791-1797

10. Schaberg T, Rebhan K, Lode H. Risk factors for side-effects of isoniazid, rifampin and pyrazinamide in patients hospital- 
ized for pulmonary tuberculosis. Eur Respir J 1996;9:20262030

11. Jain AK, Rajasekaran S, Jaggi KR, Myneedu VP. Tuberculosis of the spine. J Bone Joint Surg Am 2020;102:617-628

12. Cormican L, Hammal R, Messenger J, Milburn HJ. Current difficulties in the diagnosis and management of spinal tuberculosis. Postgrad Med J 2006;82:46-51

13. Rasouli MR, Mirkoohi M, Vaccaro AR, Yarandi KK, Rahimi-Movaghar V. Spinal tuberculosis: diagnosis and management. Asian Spine J 2012;6:294-308

14. Jain AK, Kumar J. Tuberculosis of spine: neurological deficit. Eur Spine J 2013;22 Suppl 4:624-633

15. Ali A, Musbahi O, White VLC, Montgomery AS. Spinal tuberculosis: a literature review. JBJS Rev 2019;7:e9

16. Gülbay BE, Gürkan OU, Yildiz OA, Onen ZP, Erkekol FO, Baççioğlu A, et al.. Side effects due to primary antituberculosis drugs during the initial phase of therapy in 1149 hospitalized patients for tuberculosis. Respir Med 2006;100: 1834-1842
17. Yilmaz C, Selek HY, Gürkan I, Erdemli B, Korkusuz Z. Anterior instrumentation for the treatment of spinal tuberculosis. J Bone Joint Surg Am 1999;81:1261-1267

18. Yee D, Valiquette C, Pelletier M, Parisien I, Rocher I, Menzies D. Incidence of serious side effects from first-line antituberculosis drugs among patients treated for active tuberculosis. Am J Respir Crit Care Med 2003;167:1472-1477

19. Lee JH, Park HK, Heo J, Kim TO, Kim GH, Kang DH, et al.. Drug Rash with Eosinophilia and Systemic Symptoms (DRESS) syndrome induced by celecoxib and anti-tuberculosis drugs. J Korean Med Sci 2008;23:521-525

20. Emel E, Güzey FK, Güzey D, Bas NS, Sel B, Alatas I. Non-contiguous multifocal spinal tuberculosis involving cervical, thoracic, lumbar and sacral segments: a case report. Eur Spine J 2006;15:1019-1024

21. Turgut M. Multifocal extensive spinal tuberculosis (Pott's disease) involving cervical, thoracic and lumbar vertebrae. Br J Neurosurg 2001;15:142-146 